\title{
Computing Hall subgroups of finite groups
}

\author{
Bettina Eick and Alexander Hulpke
}

\begin{abstract}
We describe an effective algorithm to compute a set of representatives for the conjugacy classes of Hall subgroups of a finite permutation or matrix group. Our algorithm uses the general approach of the so-called 'trivial Fitting model'.
\end{abstract}

\section{Introduction}

Consider the classical result of Hall that for a finite solvable group $G$ and a set $\pi$ of primes, there exists a Hall $\pi$-subgroup of $G$ and, moreover, all Hall $\pi$-subgroups of $G$ are conjugate. It is well known that Hall's result does not extend to finite groups in general. For example, the group $\mathrm{PSL}_{2}(7)$ contains two conjugacy classes of Hall $\{2,3\}$-subgroups and no Hall $\{2,7\}$ subgroup. The theory of Hall subgroups in finite groups has been studied extensively. We refer to $[\mathbf{2 4}$, Chapter 9] for a general introduction and to Revin and Vdovin $[\mathbf{2 8 , 2 9}]$ for more recent advances and further references.

For a finite solvable group, there exists a highly effective method [6] to determine a representative of the unique conjugacy class of Hall $\pi$-subgroups for any given set $\pi$ of primes. This algorithm does not extend to non-solvable finite groups. In the case of a non-solvable finite permutation or matrix group, one can compute the full subgroup lattice, or significant parts of it; see $[\mathbf{3}, \mathbf{5}]$. This allows us to determine the conjugacy classes of Hall $\pi$-subgroups as a subset of the subgroup lattice. However, this approach to computing the conjugacy classes of Hall $\pi$-subgroups seems not to be very efficient.

The aim of this paper is to introduce an effective algorithm to compute one representative for every conjugacy class of Hall $\pi$-subgroups in a finite permutation or matrix group $G$. More precisely, we solve the following problems.

- Determine all prime number sets $\pi$ for which $G$ has a Hall $\pi$-subgroup.

- Given a set $\pi$ of primes, determine the number $k_{G}(\pi)$ of conjugacy classes of Hall $\pi$-subgroups of $G$.

- Given a set $\pi$ of primes, determine a representative for every conjugacy class of Hall $\pi$-subgroups of $G$.

Our algorithm uses the structure of the given group $G$, but not its representation. It applies to finite permutation and matrix groups.

In Section 6, we will discuss the explicit determination of all conjugacy classes of Hall subgroups in a finite simple group. We recall, in Section 5, how the conjugacy classes of Hall subgroups of the simple constituents can be combined to construct the conjugacy classes of Hall subgroups of a finite group. The latter relies mainly on an effective version of the SchurZassenhaus theorem discussed in Section 4. Our main algorithm then uses the so-called trivial Fitting model, described in $\S 3.1$, to put all these ingredients together to obtain an effective algorithm for computing Hall subgroups of an arbitrary finite permutation or matrix group; see Section 7. Runtimes for the GAP implementation of our method are given in Section 8. Before embarking on the description of our algorithm, we recall some preliminaries in Sections 2 and 3.

Received 28 June 2011; revised 29 February 2012.

2010 Mathematics subject classification 20B40, 20D20, 20H20 (primary), 20-04 (secondary). 


\section{Preliminaries on Hall subgroups}

In this section we introduce our notation for Hall subgroups. Let $G$ be a finite group and $\pi$ a finite set of primes. Then $\operatorname{Hall}_{\pi}(G)$ denotes the set of all Hall $\pi$-subgroups of $G$. Denote by $\pi^{\prime}$ the set of all primes not in $\pi$. We write $|G|$ for the order of $G$ and $\|G\|$ for the set of primes dividing the order of $G$. We denote by $\pi_{G}$ the set of primes in $\pi$ which divide $|G|$; thus $\pi_{G}=\pi \cap\|G\|$. The following remark is elementary.

REMARK 1 . Let $\pi$ be a set of primes and $G$ a finite group.

(i) If $\pi_{G}=\emptyset$, then $\{1\}$ is the unique Hall $\pi$-subgroup of $G$.

(ii) If $\pi_{G}=\|G\|$, then $G$ is the unique Hall $\pi$-subgroup of $G$.

(iii) If $\pi_{G}=\{p\}$, then the Hall $\pi$-subgroups coincide with the Sylow $p$-subgroups of $G$.

The Hall subgroups $\{1\}$ and $G$ are said to be trivial. All other Hall subgroups are proper Hall subgroups. If $\pi_{G}=\{p\}$, then Hall $\pi$-subgroups exist and are all conjugate by Sylow's theorem.

The Hall subgroups of odd order often play a special role in the investigation of Hall subgroups in the literature. If they exist, they are solvable by the Feit-Thompson theorem [9] and are conjugate owing to a result by Gross [11]. Combining these two results yields the following corollary.

Corollary 2. Let $\pi$ be a set of odd primes and $G$ a finite group. If $G$ has a Hall $\pi$-subgroup, then $G$ has a Hall $\rho$-subgroup for every $\rho \subseteq \pi$.

The theory of Hall subgroups of even order differs substantially from that of Hall subgroups of odd order. We demonstrate this with the following example.

EXAmple 3 . Consider the group $G=\mathrm{PSL}_{3}(11)$ of order $2^{4} \cdot 3 \cdot 5^{2} \cdot 7 \cdot 11^{3} \cdot 19$. Then $G$ has two conjugacy classes of Hall $\{2,3\}$-subgroups and two conjugacy classes of Hall $\{2,3,5,11\}$ subgroups. Apart from its Sylow 2-subgroups and $G$ itself, the group $G$ has no further Hall subgroups of even order.

This implies that the direct product of $l$ copies of $\mathrm{PSL}_{3}(11)$ has $2^{l}$ conjugacy classes of Hall $\{2,3\}$-subgroups. Hence there is no upper bound on the number of conjugacy classes of Hall subgroups in finite groups. Further, the example shows that Corollary 2 does not extend to the even-order case.

\section{Preliminaries on algorithms}

The book [16] provides a useful introduction to computations with finite permutation and matrix groups. In this section we recall some of the algorithms for finite groups which we will use later.

\subsection{Composition tree and trivial Fitting model}

Many algorithms for finite groups proceed by induction along a subnormal series of the group under consideration. A composition series of the group is often used. The determination of a composition series is usually achieved by induction: given a finite group $G$, determine a nontrivial normal subgroup $N$ and then refine $G / N$ and $N$ further. The result of this process is recorded in the data structure of a composition tree. Its root corresponds to $G$ and has two immediate descendants which are the roots of the composition trees of $N$ and $G / N$. The leaves of the composition tree are the composition factors of $G$. We refer to $[\mathbf{1 6}, \S 10.2]$ and $[\mathbf{2 3}]$ for further details, including methods for determining a composition series in finite permutation or matrix groups. 
The trivial Fitting model uses a particular normal series for computations with the group under consideration. This model was introduced in [1], and has proved to be highly successful in the case of permutation groups; for example, it has been applied in $[\mathbf{3}, \mathbf{4}, \mathbf{1 7}]$. Its application to finite matrix groups is described in [23].

The special normal series used in the trivial Fitting model is defined as follows. Given a finite group $G$, let $R(G)$ denote the solvable radical of $G$, that is, the maximal solvable normal subgroup of $G$. Further, let $S(G) / R(G)$ be the socle of $G / R(G)$; this is a direct product of simple groups $S_{1}, \ldots, S_{n}$, say. Finally, let $T(G) / R(G)$ denote the kernel of the natural permutation action of $G / R(G)$ on the direct factors. Then the trivial Fitting model uses the series

$$
G \geqslant T(G) \geqslant S(G) \geqslant R(G) \geqslant\{1\} .
$$

This series can be determined by first computing an effective homomorphism $\rho$ from $G$ to a permutation group such that $R(G)$ is the kernel of $\rho$; see [22] for the permutation group case and [18] for the finite matrix group case. Then one can compute $S(G) / R(G)=\operatorname{Soc}(G / R(G))=$ $S_{1} \times \ldots \times S_{n}$ in the permutation group image of $\rho$ and determine the permutation action $\alpha: G / R(G) \rightarrow \operatorname{Sym}_{n}$ of $G$ on the direct factors. We refer to $[\mathbf{1 6}, \S 10.1]$ for further details.

\subsection{Sylow subgroups and their normalizers}

Cannon, Cox and Holt [2] exhibit methods for determining a Sylow p-subgroup of a permutation group and for conjugating one Sylow $p$-subgroup to another. Their approach allows determination of the normalizer of a Sylow $p$-subgroup as the stabilizer under the conjugation action. It relies on the availability of a stabilizer chain and backtrack search in the group.

An alternative approach to computing Sylow $p$-subgroups of a permutation group and their normalizers is described by Kantor in [20, 21]. It uses the structure of the underlying group and therefore applies to finite permutation and matrix groups, provided that a composition tree for the underlying group is available.

Further, we note that Stather [26] presents methods for determining Sylow subgroups and their normalizers in classical groups.

\subsection{The Frattini argument}

Suppose that a normal subgroup $N$ of a finite group $G$ is given, and let $H$ be a subgroup of $N$ such that $H^{G}=H^{N}$; that is, the $N$-conjugacy class of $H$ is fixed under the conjugation action of $G$. Then $N_{G}(H)$ is a supplement to $N$ in $G$; that is, $N_{G}(H) N=G$.

In this short subsection we point out that the group $N_{G}(H)$ can be determined using an orbit-stabilizer method in $N$. Let $G=\left\langle g_{1}, \ldots, g_{m}, N\right\rangle$. Then for every $i \in\{1, \ldots, m\}$ there exists $n_{i} \in N$ with $H^{g_{i}}=H^{n_{i}}$. Let $R=\left\langle g_{1} n_{1}^{-1}, \ldots, g_{m} n_{m}^{-1}, N_{N}(H)\right\rangle$. Thus generators for $R$ can be determined from the given generators for $G$ using calculations in $H$. It is elementary to observe that

$$
R=N_{G}(H) .
$$

This method applies, for example, when $H$ is a Hall subgroup of the normal subgroup $N$ and $\operatorname{gcd}(|N|,[G: N])=1$.

\section{Coprime complements}

The Schur-Zassenhaus theorem [24, Theorem 9.2.1] asserts that given a normal subgroup $N$ in a finite group $G$ such that $\operatorname{gcd}(|N|,[G: N])=1$, there exists a unique conjugacy class of complements to $N$ in $G$. The aim of this section is to describe an effective algorithm for determining a complement in this situation. This algorithm will be a key component in the methods we develop later. 
Our algorithm distinguishes between the cases of $N$ being solvable and non-solvable. Before we consider these two cases, we introduce a general reduction which is usually very fast and effective.

\subsection{A preliminary reduction step}

Let $G$ be a finite group and $N$ a normal subgroup of $G$ with $\operatorname{gcd}(|N|,[G: N])=1$. Let $\pi$ be the set of prime divisors of $[G: N]$ and let $\pi^{\prime}:=\|N\|$. For a natural number $x$, we write $x \in \pi$ (respectively, $x \in \pi^{\prime}$ ) if every prime divisor of $x$ is in $\pi$ (respectively, $\pi^{\prime}$ ).

Let $g \in G$ be of order $o$. Then $o=x y$ with $x \in \pi$ and $y \in \pi^{\prime}$. Using the extended gcd-algorithm, we determine $a, b \in \mathbb{Z}$ with $1=a x+y b$. Then

$$
g=g^{1}=g^{a x+y b}=g^{a x} g^{b y},
$$

and thus we can write $g$ as a product of the commuting elements $g^{a x}$ and $g^{b y}$, where $g^{a x}$ has order $y$ and $g^{b y}$ has order $x$. By construction, it follows that $g^{b y} \in N$. We define $\bar{g}=g^{a x}$.

Lemma 4. Let $G$ be a finite group and let $N \unlhd G$ with $\operatorname{gcd}(|N|,[G: N])=1$. Let $g_{1}, \ldots, g_{m} \in G$ be a generating set of $G$ modulo $N$; that is, $G=\left\langle g_{1}, \ldots, g_{m}, N\right\rangle$. Let $R=$ $\left\langle\overline{g_{1}}, \ldots, \overline{g_{m}}\right\rangle$ and $M=R \cap N$.

(a) There exists a unique conjugacy class of complements to $M$ in $R$. Any complement to $M$ in $R$ is a complement to $N$ in $G$ as well.

(b) For any complement $C$ to $N$ in $G$ we have that $R \leqslant[N, C]^{N} \cdot C$. In particular, if $N$ is central in $G$, then $R$ is a complement to $N$ in $G$.

Proof. Assertion (a) follows directly, as $R$ covers $G / N$ and thus $[R: M]$ is coprime to $M$.

(b) Suppose that $C$ is an arbitrary complement to $N$ in $G$ and let $g \in G$. Then $g=c n$ with $c \in C$ and $n \in N$, and thus for $k \in \mathbb{N}$ we find that

$$
\begin{aligned}
g^{k} & =(c n)^{k}=c^{2} n^{c} n(c n)^{k-2}=\ldots=c^{k} n^{c^{k-1}} n^{c^{k-2}} \ldots n^{c} n \\
& =c^{k} n^{k}\left[n, c^{k-1}\right]^{n^{k-2}}\left[n, c^{k-2}\right]^{n^{k-3}} \ldots\left[n, c^{2}\right]^{n}[n, c] .
\end{aligned}
$$

As above, let $o$ be the order of $g$, write $o=x y$ with $x \in \pi$ and $y \in \pi^{\prime}$, and let $1=a x+b y$ be the result of the extended gcd-computation. Then for $k=a x$, we find that $c^{a x}=1$ and thus

$$
g^{a x}=n^{c^{k-1}} n^{c^{k-2}} \ldots n^{c} n \in N .
$$

For $k=b y$ we get that

$$
\begin{aligned}
g^{b y} & =c^{k} n^{k}\left[n, c^{k-1}\right]^{n^{k-2}}\left[n, c^{k-2}\right]^{n^{k-3}} \ldots\left[n, c^{2}\right]^{n}[n, c] \\
& =c\left[n, c^{k-1}\right]^{n^{k-2}}\left[n, c^{k-2}\right]^{n^{k-3}} \ldots\left[n, c^{2}\right]^{n}[n, c],
\end{aligned}
$$

using that $c^{b y}=c$ and $n^{y}=1$. Thus $\bar{g}$ differs from $c$ by an element of the normal closure of $[N, C]$.

\subsection{The case where $N$ is solvable}

Let $G$ be a finite group with a solvable normal subgroup $N$ such that $\operatorname{gcd}(|N|,[G: N])=1$.

If $N$ is an elementary abelian $p$-group, then a complement to $N$ in $G$ can be computed effectively via computation of a first cohomology group. The latter reduces mainly to solving a system of linear equations over the field with $p$ elements. We refer to $[\mathbf{7}, \mathbf{1 6}]$ for details.

In the general case where $N$ is solvable but not necessarily elementary abelian, a complement to $N$ in $G$ can be determined by using induction along a $G$-normal series with elementary abelian factors. Each induction step can be facilitated by the method for the elementary abelian normal subgroup. The Schur-Zassenhaus theorem implies that a unique conjugacy class of complements exists in each induction step, and hence this approach yields a complement to $N$ in $G$. 
The following remark summarizes this.

REMARK 5. Let $G$ be a finite group and let $N$ be a solvable normal subgroup of $G$ with $\operatorname{gcd}(|N|,[G: N])=1$. Then there exists an effective algorithm for computing a representative of the unique conjugacy class of complements to $N$ in $G$.

We note that this method is also applicable to factor groups; that is, for $K \triangleleft N \triangleleft G$ it can be used to determine subgroups $K \leq C \leq G$ such that $C / K$ is a complement to $N / K$ in $G / K$. For this, we simply represent elements of $G / K$ by representatives in $G$ but test for equality of elements modulo $K$.

\subsection{The case where $N$ is non-solvable}

Let $G$ be a finite group with an arbitrary normal subgroup $N$ such that $\operatorname{gcd}(|N|,[G: N])=1$. We determine a complement to $N$ in $G$ using a method based on the following elementary lemma.

Lemma 6. Let $G$ be a finite group and let $N \unlhd G$ with $\operatorname{gcd}(|N|,[G: N])=1$. Let $p$ be a prime divisor of $|N|$, let $P$ be a Sylow $p$-subgroup of $N$, and let $C$ be a non-trivial characteristic subgroup of $P$. Then $C^{G}=C^{N}$ and $G=N N_{G}(C)$.

Proof. Let $g \in G$. Then $P^{g}=P^{n}$ for some $n \in N$. Thus $g n^{-1}$ normalizes $P$ and hence also $C$. Thus $C^{g}=C^{n}$ follows. The Frattini argument now yields the desired result.

Lemma 6 implies that a complement to $N$ in $G$ can be obtained as follows:

- choose $p$ and determine $P$ (as in $\S 3.2$ );

- choose $C$ as in Lemma 6;

- determine $R=N_{G}(C)$ (as in $\S 3.3$ );

- determine a complement $H / C$ to $(R \cap N) / C$ in $R / C$;

- determine a complement $K$ to $C$ in $H$;

- $K$ is then a complement to $N$ in $G$.

If $N$ is non-solvable, then 2||$N \mid$. Hence we can choose $p=2$. Let $C=P$ in Lemma 6 . Then $R / C$ is a group of odd order and hence solvable. Thus $H / C$ can be obtained using the method of $\S$ 4.2. Further, the group $C$ is solvable and hence $K$ can be obtained using the method of $\S 4.2$. In summary, we obtain that a complement to $N$ in $G$ can be determined using two applications of the algorithm in $\S 4.2$.

\subsection{Summary}

We summarize our approach to computing a complement to $N$ in $G$ in the case where $G$ is a finite group, $N$ is a normal subgroup of $G$ and $\operatorname{gcd}(|N|,[G: N])=1$.

Step 1. Determine the subgroup $R$ as in Lemma 4 and replace $G$ by $R$ and $N$ by $N \cap R$. Note that $N \cap R$ can be computed as an intersection or as the kernel of a homomorphism.

Step 2. Check whether $N$ is solvable. If not, then employ the method of $\S 4.3$; if so, then use the method of $\S 4.2$.

\section{Reduction via normal subgroups}

Let $G$ be a finite group and let $N$ be an arbitrary normal subgroup of $G$. Suppose that sets of representatives for the conjugacy classes of Hall $\pi$-subgroups in $N$ and in $G / N$ are given. In this section we present an effective algorithm for determining, from this information, a set of representatives for the conjugacy classes of Hall $\pi$-subgroups in $G$. Iterated application 
of this method eventually reduces the computation of conjugacy class representatives of Hall $\pi$-subgroups in $G$ to finding the simple composition factors of $G$.

Our first lemma motivates this reduction approach. It implies that if a finite group $G$ has a Hall $\pi$-subgroup, then every composition factor of $G$ also has a Hall $\pi$-subgroup. See [14, Lemma 1] for a proof.

Lemma 7 (Hall). Let $G$ be a finite group with normal subgroup $N$ and let $\pi$ be a set of primes. If $H \in \operatorname{Hall}_{\pi}(G)$, then $H N / N \in \operatorname{Hall}_{\pi}(G / N)$ and $H \cap N \in \operatorname{Hall}_{\pi}(N)$.

Now suppose that every composition factor of the finite group $G$ has a Hall $\pi$-subgroup. The following elementary lemma gives a criterion for when these Hall $\pi$-subgroups can be combined to give a Hall $\pi$-subgroup of the full group $G$. We include a proof for completeness.

Lemma 8. Let $G$ be a finite group, let $N \unlhd G$, let $K \in \operatorname{Hall}_{\pi}(N)$ and let $L / N \in \operatorname{Hall}_{\pi}(G / N)$.

(a) There exists $H \in \operatorname{Hall}_{\pi}(G)$ with $H \cap N=K$ and $H N=L$ if and only if for every $l \in L$ there exists $n \in N$ with $K^{n}=K^{l}$.

(b) Let $H_{1}, H_{2} \in \operatorname{Hall}_{\pi}(G)$ with $H_{i} \cap N$ conjugate to $K$ in $N$ and $\left(H_{i} N\right) / N$ conjugate to $L / N$ in $G / N$. Then $H_{1}$ and $H_{2}$ are conjugate in $G$.

Proof. (a) If $H$ exists, then it normalizes $K$ and hence $L=H N$ normalizes the $N$-conjugacy class of $K$. It remains to consider the converse. Thus, suppose that $L$ normalizes the $N$ conjugacy class of $K$. Our aim is to construct a Hall $\pi$-subgroup $H$ with $H \cap N=K$ and $H N=L$. First, we determine $M=N_{L}(K)$. The Frattini argument implies that $M N=L$. Hence $H / K$ is a complement to $(M \cap N) / K$ in $M / K$. Such a complement exists by the Schur-Zassenhaus theorem [24, Theorem 9.1.2].

(b) Using the conjugation in $N$ and in $G / N$, we can assume that $H_{1} \cap N=K=H_{1} \cap N$ and $H_{1} N=H_{2} N=L$. Next, we observe that both $H_{1}$ and $H_{2}$ are complements to $N / K$ in $N_{L}(K)$. By the Schur-Zassenhaus theorem [24, Theorem 9.1.2], there exists at most one conjugacy class of complements of this type. Hence $H_{1}$ is conjugate to $H_{2}$ in $N_{L}(K)$ and thus in $G$.

Let $G$ be a finite group with a normal subgroup $N$. Suppose that we have determined the conjugacy classes of Hall $\pi$-subgroups in $N$ and in $G / N$. To determine the conjugacy classes of Hall $\pi$-subgroups of $G$, we consider a representative $K$ for each class in $N$ and a representative $L / N$ for each class in $G / N$ and do the following:

(1) check whether $K^{N}=K^{L}$; if not, then report failure;

(2) determine $M=N_{L}(K)$ (using $\S 3.3$ );

(3) determine a complement $H / K$ to $(M \cap N) / K$ in $M / K$ (using Section 4);

(4) return $H$.

For step (1), note that $N \leq L$. Thus $K^{N}=K^{L}$ holds if and only if for each generator $l$ of $L$ there is an element $n \in N$ such that $K^{l}=K^{n}$ holds. Checking the latter requires a conjugacy test for Hall $\pi$-subgroups of $N$. If $N$ is abelian, Hall $\pi$-subgroup conjugation is trivial; otherwise, this conjugacy test can be performed by representing $N$ as an intransitive permutation group (with orbits corresponding to direct factors) and performing a backtrack search iterated over all direct factors. We note that in our later applications, $N$ will be minimally normal and thus elementary.

In step (3) we are using a complement computation in a factor group, as described after Remark 5. Thus this complement is automatically obtained as a subgroup $H \geq K$.

\section{Hall subgroups of simple groups}

Much is known about the Hall subgroups of the finite simple groups. In this section we give a brief overview of this. Before doing so, we recall the following useful result due to Revin and 
Vdovin [28] and Gross [11] on the number of conjugacy classes of Hall subgroups in finite simple groups.

Theorem 9 (Gross, Revin and Vdovin). Let $G$ be a finite simple group.

(a) If $2 \notin \pi$, then $k_{G}(\pi) \in\{0,1\}$.

(b) If $3 \notin \pi$, then $k_{G}(\pi) \in\{0,1,2\}$.

(c) If $2,3 \in \pi$, then $k_{G}(\pi) \in\{0,1,2,3,4,9\}$.

Further, we also recall a theorem of Gross [11] on Hall subgroups of odd order in finite simple groups.

Theorem 10 (Gross). Let $H$ be a Hall $\pi$-subgroup of the finite simple group $G$ and suppose that $H$ has odd order. Then $H$ has a Sylow tower; that is, there is a series of normal subgroups $H=H_{1} \geqslant H_{2} \geqslant \ldots \geqslant H_{n} \geqslant\{1\}$ in $H$ such that $H_{i} / H_{i+1}$ is isomorphic to a Sylow subgroup of $H$ (and of $G$ ).

We note that not only is the existence of a Sylow tower known but also the ordering of the primes underlying it. We refer to [11] for details.

\subsection{The alternating groups}

The Hall subgroups of the alternating groups are well understood. Apart from the trivial cases and the Sylow subgroups, they are surprisingly rare. We recall the following result of Hall [14] and Thompson [30].

Theorem 11 (Hall and Thompson). Let $\pi$ be a set of primes and let $n \in \mathbb{N}$ with $n \geqslant 5$. Then $\mathrm{Alt}_{n}$ has at most one conjugacy class of Hall $\pi$-subgroups; it has exactly one such conjugacy class if and only if one of the following holds.

(a) The Hall $\pi$-subgroup is either trivial or a Sylow subgroup; this is the case when $\pi \cap\left\|\operatorname{Alt}_{n}\right\|=\emptyset, \pi \cap\left\|\operatorname{Alt}_{n}\right\|=\{p\}$ or $\left\|\operatorname{Alt}_{n}\right\| \subseteq \pi$.

(b) Alt $_{n-1}$ is a Hall $\pi$-subgroup; this is the case when $n$ is a prime and $\pi$ contains all primes less than $n$ but not $n$ itself.

(c) $\pi \cap\left\|\operatorname{Alt}_{n}\right\|=\{2,3\}$ and $n \in\{7,8\}$.

In the two exceptional cases of Theorem 11(c), it is not difficult to determine the Hall subgroups explicitly. They are groups of order 72 and 576 , respectively.

\subsection{The sporadic groups}

For the 26 sporadic groups, there are tables of those sets $\pi$ of primes available for which a Hall $\pi$-subgroup exists. For Hall subgroups of odd order, these have been determined by Gross [13]. For the case where $2 \in \pi$, we refer to Revin and Vdovin [27].

As a result, it is known that 17 of the 26 sporadic groups have non-trivial and non-Sylow Hall subgroups. Table 1 lists their corresponding sets $\pi$. A superscript 2 on a set $\pi$ of primes indicates that there are two conjugacy classes of Hall $\pi$-subgroups. If no superscript is present, then there is just one class. An explicit isomorphism test shows that two subgroups from different conjugacy classes are always non-isomorphic.

\subsection{The simple groups of Lie type}

Theorem 10 asserts that an odd-order Hall subgroup in a simple group has a Sylow tower. This can be refined for the simple groups of Lie type, as the following results of Vdovin and Revin [27] and Gross [11] show. 
TheOrem 12. Let $G$ be a finite simple group of Lie type over a field of characteristic $p$. Let $H$ be a Hall $\pi$-subgroup of $G$ with $2 \notin \pi$.

(a) (Vdovin and Revin) Suppose $p \notin \pi$. Let $r$ be the least prime in $\pi$ and let $\rho=\pi \backslash\{r\}$. Then $H$ has a normal abelian Hall $\rho$-subgroup.

(b) (Gross) Suppose $p \in \pi$. Let $\rho=\pi \backslash\{p\}$. Then $H$ has a normal Hall $\rho$-subgroup.

The even-order Hall subgroups of the simple groups of Lie type are very complex and technically difficult to describe. Vdovin and Revin [27] assert that they have achieved a complete classification.

Here we consider only the frequently occurring case $\mathrm{PSL}_{2}(q)$ in more detail. In this case, there exists a complete classification of all subgroups that is due to Dickson; see also [19, p. 213]. This can be used to obtain a classification of Hall subgroups. We recall Dickson's classification briefly in the following theorem. Observe that $\left|\mathrm{PSL}_{2}(q)\right|=(q-1) q(q+1) \cdot \operatorname{gcd}(q, 2) / 2$, and denote by $\pi_{-}$the odd prime divisors of $q-1$ and by $\pi_{+}$the odd prime divisors of $q+1$. Note that $\pi_{+} \cap \pi_{i}=\emptyset$.

Theorem 13. Let $q=p^{f}$ be a power of the prime $p$. Then the group $G=\operatorname{PSL}_{2}(q)$ has exactly the following classes of non-trivial Hall $\pi$-subgroups with $\pi_{G}=\pi \cap\|G\|$ :

(a) one class of subgroups for any set $p \in \pi_{G} \subseteq\{p\} \cup \pi_{-}$;

(b) one class of subgroups for any subset $\pi_{G} \subseteq \pi_{+}$or $\pi_{G} \subseteq \pi_{-}$;

(c) if $q-1 \equiv 2(\bmod 4)$, one class of subgroups for $\pi_{G}=\{2\} \cup \omega$ with $\omega \subseteq \pi_{+}$;

(d) if $q-1 \equiv 0(\bmod 4)$, one class of subgroups for $\pi_{G}=\{2\} \cup \omega$ with $\omega \subseteq \pi_{-}$;

(e) if the $\{2,3\}$-part (respectively, the $\{2,3,5\}$-part) of $|G|$ is exactly 12,24 or 60 , subgroups of type $\mathrm{Alt}_{4}, \mathrm{Sym}_{4}$ and $\mathrm{Alt}_{5}$ happen to occur as Hall $\pi_{G}$-subgroups.

Proof. Let $H$ be a non-trivial Hall $\pi_{G}$-subgroup of $G$ for a subset $\pi_{G} \subseteq\|G\|$. Then $H$ must be in one of the seven classes of subgroups in [19, Hauptsatz 8.27, p. 213], denoted by (1)-(7). We shall show that $H$ is described by the classes (a)-(e) in our theorem.

Assume first that $p|| H \mid$; that is, in the classification of subgroups we consider classes (1) and (7). Then $H$ contains a $p$-Sylow subgroup which is (up to conjugacy in $G$ ) a group $U(2, q)$ of upper triangular matrices with diagonal entries 1. The classification from [19, Hauptsatz 8.27, p. 213] shows that any proper subgroup of $G$ containing $U(2, q)$ is contained in the group $B(2, q)$ of upper triangular matrices. The group $B(2, q)$ is solvable and thus has exactly one conjugacy

TABLE 1. Hall subgroups for sporadic simple groups.

\begin{tabular}{ll}
\hline Group & Sets $\pi$ \\
\hline $\mathrm{M}_{11}$ & $\{5,11\},\{2,3\},\{2,3,5\}$ \\
$\mathrm{M}_{12}$ & $\{5,11\}$ \\
$\mathrm{M}_{22}$ & $\{5,11\},\{2,3,5\}$ \\
$\mathrm{M}_{23}$ & $\{5,11\},\{11,23\},\{2,3\},\{2,3,5\}^{2},\{2,3,5,7\}^{2},\{2,3,5,7,11\}$ \\
$\mathrm{M}_{24}$ & $\{5,11\},\{11,23\},\{2,3,5\}$ \\
$\mathrm{J}_{1}$ & $\{3,5\},\{3,7\},\{3,19\},\{5,11\},\{2,3\},\{2,7\},\{2,3,5\},\{2,3,7\}$ \\
$\mathrm{J}_{4}$ & $\{5,7\},\{5,11\},\{5,31\},\{7,29\},\{7,43\},\{2,3,5\}$ \\
.1 & $\{11,23\}$ \\
.2 & $\{11,23\}$ \\
.3 & $\{11,23\}$ \\
$\mathrm{Ly}$ & $\{11,67\}$ \\
$\mathrm{Ru}$ & $\{7,29\}$ \\
$\mathrm{ON}$ & $\{3,5\},\{5,11\},\{5,31\}$ \\
$\mathrm{M}(23)$ & $\{11,23\}$ \\
$\mathrm{M}(24)^{\prime}$ & $\{11,23\}$ \\
$\mathrm{F}_{2}$ & $\{11,23\},\{23,47\}$ \\
$\mathrm{F}_{1}$ & $\{23,47\},\{29,59\}$ \\
\hline
\end{tabular}


class of Hall $\pi$-subgroups for $\pi=\|H\|$; let $U$ denote one such subgroup. Then $H$ is conjugate in $B(2, q)$ (and thus in $G$ ) to $U$. As $[G: B(2, q)]=1+q$, we know that $\pi_{G} \subseteq\{p\} \cup \pi_{-} \cup\{2\}$. But if $q$ is odd, then $1+q$ is even; and if $2 \in \pi_{G}$, then $H$ would not be a Hall subgroup of $G$. Thus, for odd $p$, we know that $2 \notin \pi_{G}$ and we get in general that $p \in \pi_{G} \subseteq\{p\} \cup \pi_{-}$. Thus all Hall $\pi_{G^{-}}$ subgroups described in this section are of type (a). Conversely, for any set $p \in \pi_{G} \subseteq\{p\} \cup \pi_{-}$, such Hall $\pi_{G}$-subgroups exist as Hall subgroups of $B(2, q)$.

We now consider Hall subgroups $H$ such that $p X|H|$. The class (2) of subgroups of $\mathrm{PSL}_{2}(q)$ consists of cyclic subgroups of order dividing $s:=(q+1) / \operatorname{gcd}(2, q-1)$ or $t:=(q-$ $1) / \operatorname{gcd}(2, q-1)$. A cyclic subgroup of $G$ of order $s$ is a Singer cycle and is a $\pi_{+}$-subgroup of $G$. Being solvable, this Singer cycle has Hall subgroups for any subset $\pi_{G} \subseteq \pi_{+}$. All of these are Hall subgroups of $G$. By [13], there is only one class of such subgroups in $G$. These are subgroups of type (b) for subsets of $\pi_{+}$.

The normalizer in $G$ of the Singer cycle is dihedral of order $2 \cdot(q+1) / \operatorname{gcd}(2, q-1)$ and thus gives rise to dihedral subgroups (of class (3)) of order $2 \cdot a$ for every divisor $a$ of $s$ (which are in one-to-one correspondence with the subgroups of the Singer cycle, so that there is one $G$-conjugacy class each). If $q-1 \equiv 2(\bmod 4)$ and $a$ is odd, these dihedral subgroups will be Hall $\pi_{G}$-subgroups for $\pi_{G}=\{2\} \cup \omega$ where $\omega \subseteq \pi_{+}$. This yields the subgroups of type (c).

For the rest of class (2), the subgroup of diagonal elements is cyclic of order $t$ and thus provides one class of Hall $\pi_{G}$-subgroups for any $\pi_{G} \subseteq \pi_{-}$. All of these are also Hall subgroups of $G$ (of type (b)). Again, [13] implies that there is only one $G$-class of these subgroups.

As above (and with the same arguments for one $G$-class of subgroups), this cyclic group of diagonal elements has a normalizer that is larger by a factor of 2 and which gives rise to dihedral subgroups in class $(3)$. For $q-1 \equiv 0(\bmod 4)($ implying $q+1 \equiv 2(\bmod 4))$, they are Hall $\pi_{G}$-subgroups for $\pi_{G}=\{2\} \cup \omega$ where $\omega \subseteq \pi_{-}$. These are of type (d).

This covers all classes of subgroups in [19, Hauptsatz 8.27, p. 213], apart from classes (4)-(6). These are subgroups of type $\mathrm{Alt}_{4}, \mathrm{Sym}_{4}$ or Alt 5 and are thus of type (e) in our listing.

While a similar approach is possible for higher Lie rank, the situation quickly becomes complicated. For example, as Table 2 in $\S 6.5$ shows, there are no non-trivial Hall subgroups in $\mathrm{PSL}_{4}(2)$, but many in $\mathrm{PSL}_{5}(2)$. This includes a Hall $\{3,7\}$-subgroup, despite the fact that neither prime arises in $\mathrm{PSL}_{5}(2): \mathrm{PSL}_{4}(2)$.

\subsection{An algorithmic approach}

Let $G$ be a finite group and let $\pi$ be a set of primes. In this section we describe a method to determine representatives for the conjugacy classes of Hall $\pi$-subgroups in $G$ without reducing to normal subgroups or factor groups. The approach is therefore aimed primarily at simple groups. To avoid trivialities, we assume that $\pi_{G} \neq \emptyset$ and $\pi_{G} \neq\|G\|$.

The basic observation is that a Hall $\pi$-subgroup will contain Sylow $p$-subgroups for every $p \in \pi$. Hall subgroups can therefore be built iteratively from their Sylow subgroups. Let $\operatorname{Syl}_{p}(G)$ denote the set of all Sylow $p$-subgroups of $G$. For any set of primes $\omega$, let $\mathcal{A}(\omega, G)$ denote a set of representatives for the conjugacy classes of subgroups of $G$ of the form $\left\langle S_{p} \mid p \in \omega, S_{p} \in \operatorname{Syl}_{p}(G)\right\rangle$.

Lemma 14. Let $\pi$ be a set of primes and $G$ a finite group. Choose $p \in \pi$ and let $P \in \operatorname{Syl}_{p}(G)$ and $\omega=\pi \backslash\{p\}$. Then

$$
\mathcal{A}(\pi, G) \subseteq\left\{\left\langle A, P^{g}\right\rangle \mid A \in \mathcal{A}(\omega, G), g \in T_{A}\right\},
$$

where $T_{A}$ is a set of representatives for the double cosets $N_{G}(P) \backslash G / N_{G}(A)$.

Proof. Let $B \in \mathcal{A}(\pi, G)$, and let $U$ be the subgroup generated by the $q$-Sylow subgroups of $B$ for $q \in \omega$. Then $U=A^{x}$ for some $x \in G$, where $A \in \mathcal{A}(\omega, G)$ and $B=\langle U, V\rangle$ for some $V=P^{y}$ 
with $y \in G$. Thus $B$ is conjugate to $B^{x^{-1}}=\left\langle A, P^{y x^{-1}}\right\rangle$. Let $g \in T_{A}$ with $a g b=y x^{-1}$ for some $a \in N_{G}(P)$ and $b \in N_{G}(A)$. Then $\left\langle A, P^{a g b}\right\rangle=\left\langle A, P^{g b}\right\rangle$ is conjugate to $\left\langle A^{b^{-1}}, P^{g}\right\rangle=\left\langle A, P^{g}\right\rangle$. Thus $B$ is conjugate to $\left\langle A, P^{g}\right\rangle$, as desired.

Every Hall $\pi$-subgroup $H$ of the finite group $G$ is conjugate to a group in $\mathcal{A}(\pi, G)$. Hence our approach to computing conjugacy class representatives for the Hall $\pi$-subgroups in $G$ is by determining

$$
\{H \in \mathcal{A}(\pi, G) \mid\|H\| \subseteq \pi\} .
$$

The following steps summarize our approach to computing a set of representatives for the conjugacy classes of Hall $\pi$-subgroups in a finite group $G$.

(1) Initialize $\mathcal{S}=\{\langle 1\rangle\}$.

(2) For $p \in \pi$ do (iterate steps 3-9).

(3) Set $\mathcal{T}=\{\}$.

(4) Determine $P \in \operatorname{Syl}_{p}(G)$ and $N=N_{G}(P)$ using $\S \S 3.2$ and 3.3 .

(5) For $A \in \mathcal{S}$ do (iterate steps 6-8).

(6) Compute the double coset representatives $T_{A}$ using [25].

(7) For $g \in T_{A}$ do (iterate step 8).

(8) Let $C=\left\langle A, P^{g}\right\rangle$. If $\|C\| \subseteq \pi$ and $C$ is not $G$-conjugate to a subgroup in $\mathcal{T}$, then add $C$ to $\mathcal{T}$.

(9) Reset $\mathcal{S}=\mathcal{T}$.

Usually, most of the groups $C$ in step (8) are not $\pi$-subgroups. It is therefore preferable to first perform a series of cheap tests for prime factors of $|C|$ before determining the order. Suitable tests are, for example, orders of random elements and lengths of orbits. These tests often eliminate candidate groups $C$ without ever having to determine their exact order.

A straightforward modification of this procedure can determine the Hall subgroups for several or all possible sets $\pi$.

TABLE 2. Hall subgroups for the simple groups of the Lie type of order at most $2 \times 10^{9}$, excluding groups of type $\mathrm{PSL}_{2}$.

\begin{tabular}{lrl}
\hline Group $G$ & Order of $G$ & Sets $\pi$ of primes \\
\hline $\mathrm{PSL}_{(}(3)$ & 5616 & $\{2,3\}^{2 a 1}$ \\
$\mathrm{Sz}(8)$ & 29120 & $\{2,7\}$ \\
$\mathrm{PSU}_{3}(4)$ & 62400 & $\{2,3\},\{3,5\},\{3,13\}$ \\
$\mathrm{PSL}_{3}(5)$ & 372000 & $\{2,3\}^{2 a 2},\{3,31\},\{2,3,5\}^{2 a 1}$ \\
$\mathrm{PSp}_{4}(4)$ & 979200 & $\{2,3\}$ \\
$\mathrm{PSp}_{4}(5)$ & 4680000 & $\{2,3\}$ \\
$\mathrm{PSU}_{3}(8)$ & 5515776 & $\{2,7\}$ \\
$\mathrm{PSU}_{3}(7)$ & 5663616 & $\{2,3\},\{3,7\},\{3,43\}$ \\
$\mathrm{PSL}_{5}(2)$ & 9999360 & $\{2,3\}^{3 a 2},\{3,7\},\{5,31\},\{2,3,7\}^{2 a 1},\{2,3,5,7\}^{2 a 1}$ \\
$\mathrm{PSU}_{5}(2)$ & 13685760 & $\{5,11\}$ \\
$\mathrm{PSL}_{3}(8)$ & 16482816 & $\{2,7\},\{2,3,7\}^{2 a 1}$ \\
$\mathrm{Sz}_{32}(32$ & 32537600 & $\{2,31\}$ \\
$\mathrm{PSL}_{3}(9)$ & 42456960 & $\{7,13\},\{2,3,5\}^{2 a 1}$ \\
$\mathrm{PSU}_{3}(11)$ & 70915680 & $\{2,3\},\{5,11\}$ \\
$\mathrm{PSp}_{4}(7)$ & 138297600 & $\{2,3\}$ \\
$\mathrm{O}_{8}^{-}(2)$ & 197406720 & $\{2,3,5\}$ \\
$\mathrm{PSL}_{3}(11)$ & 212427600 & $\{2,3\}\}^{2 a 2},\{3,5\},\{3,19\},\{7,19\},\{3,7\},\{5,11\}$, \\
& & $\{3,7,19\},\{2,3,5,11\}^{2 a 1}$ \\
$\mathrm{PSL}_{3}(13)$ & 270178272 & $\{2,3\}$ \\
$\mathrm{PSU}_{3}(13)$ & 811273008 & $\{2,3\}$ \\
$\mathrm{PSU}_{4}(4)$ & 1018368000 & $\{2,3\}$ \\
$\mathrm{PSp}_{4}(8)$ & 1056706560 & $\{2,7\},\{5,73\},\{3,157\},\{3,13\}$ \\
$\mathrm{PSL}_{3}(16)$ & 1425715200 & $\{2,5\},\{3,5\},\{7,13\}$ \\
& &
\end{tabular}




\subsection{Application}

The algorithm of $\S 6.4$ has been used to determine the Hall subgroups of all simple groups of Lie type (excluding $\mathrm{PSL}_{2}$ ) up to order $2 \times 10^{9}$. We record the results Table 2. For each group $G$ considered, this table states the order of $G$ and lists the sets $\pi$ of primes for which Hall $\pi$ subgroups exist. A superscript $k a x$ on a set $\pi$ of primes indicates that there are $k$ conjugacy classes of Hall $\pi$-subgroups in the group $G$ and that these fuse to $x$ classes under the action of $\operatorname{Aut}(G)$. For the groups $G$ in this table, an explicit isomorphism test shows that in the case of $x>1$, two subgroups from different $\operatorname{Aut}(G)$-classes are not isomorphic.

\section{The trivial Fitting model}

Sections 4-6 can now readily be combined to produce a first algorithm for determining Hall subgroups: this computes a composition series of $G$, determines the Hall subgroups up to conjugacy for each simple factor of $G$, and then combines them using Sections 5 and 4 . A slight improvement can be obtained if a chief series is used instead of a composition series.

In this section we improve this approach further by using the trivial Fitting model of $\S 3.1$. This model can be used to obtain an effective solution of the central problems treated in this paper, as stated in Section 1.

\subsection{Computing Hall subgroups}

Suppose that a finite permutation or matrix group $G$ is given, together with a set $\pi$ of primes. The aim is to determine a representative for every conjugacy class of Hall $\pi$-subgroups in $G$. As a first step, we determine the normal series used in the trivial Fitting model,

$$
G \geqslant T \geqslant S \geqslant R \geqslant\{1\}
$$

Let $\rho$ be the corresponding effective homomorphism from $G$ to a permutation group, with $R=$ ker $\rho$. Let $S / R=S_{1} \times \ldots \times S_{n}$ with $S_{i}$ simple, and let $\alpha: G / R \rightarrow \operatorname{Sym}_{n}$ be the permutation action of $G / R$ on the direct factors of $S / R$ so that $T / R$ is the kernel of $\alpha$.

The quotient $G / T$ has a natural permutation representation of degree $n$. We assume, by induction on the order of the group, that we are given a set of conjugacy class representatives $H_{1} / T, \ldots, H_{r} / T$ of the Hall $\pi$-subgroups of $G / T$.

The subquotient $T / S$ is solvable by Schreier's conjecture [8] and has a permutation representation of bounded degree; see $[\mathbf{1 5}, \mathbf{2 2}]$. A representative $K / S$ of the single conjugacy class of Hall $\pi$-subgroups in $T / S$ can be computed as in [6].

The subquotient $S / R$ is a direct product of simple groups $S_{1}, \ldots, S_{n}$. Note that $K$ acts on each $S_{i}$ by conjugation. Using Section 6 , we determine representatives for the $K$-invariant conjugacy classes of Hall $\pi$-subgroups for each $S_{i}$. We then use their direct products to obtain representatives $M_{1} / R, \ldots, M_{l} / R$ for the $K$-invariant conjugacy classes of Hall $\pi$-subgroups in $S / R$.

The group $R$ is solvable, and a representative $J$ for its unique conjugacy class of Hall $\pi$-subgroups can be computed with the methods of [6].

We now combine the Hall $\pi$-subgroups obtained for $G / T, T / S, S / R$ and $R$ to obtain Hall $\pi$-subgroups of $G$. To this end, for each $i \in\{1, \ldots, r\}$ we consider the subgroup $H_{i} / T$ of $G / T$ and perform the following tasks.

(1) Compute a subgroup $L_{i} / S$ with $L_{i} T=H_{i}$ and $L_{i} \cap T=K$, using Section 4 .

(2) Determine $I_{i} \subseteq\{1, \ldots, l\}$ so that the $S / R$-conjugacy class of $M_{j} / R$ is invariant under the conjugation action of $L_{i} / R$ if and only if $j \in I_{i}$.

(3) For each $j \in I_{i}$ do:

(a) compute $N_{i j} / R$ with $N_{i j} S=L_{i}$ and $\left(N_{i j} \cap S\right) / R=M_{j}$, using Section 4 ;

(b) compute $W_{i j}$ with $W_{i j} R=N_{i j}$ and $\left(W_{i j} \cap R\right)=J$, using Section 4.

(4) Return $\left\{W_{i j} \mid j \in I_{i}\right\}$. 
We note that the computed subgroups in steps (1), (3a) and (3b) exist by Lemma 8. Also, using Lemma 8, it is not difficult to show that the set of subgroups

$$
\left\{W_{i j} \mid 1 \leqslant i \leqslant n, j \in I_{i}\right\}
$$

thus determined is a complete and irredundant set of conjugacy class representatives for the Hall $\pi$-subgroups of $G$.

\subsection{Determining the number of conjugacy classes}

Suppose that a finite permutation or matrix group $G$ is given, together with a set $\pi$ of primes. The aim is to determine the number of conjugacy classes of Hall $\pi$-subgroups of $G$.

It is straightforward to modify the algorithm of $\S 7.1$ for this purpose. In fact, it is sufficient to determine the Hall $\pi$-subgroups of $G / T, T / S$ and $S / R$ and then perform only steps (1) and (2) of the algorithm. The number of conjugacy classes is then

$$
\sum_{i=1}^{n}\left|I_{i}\right| .
$$

\subsection{Finding all sets of primes}

Suppose that a finite permutation or matrix group $G$ is given. The aim is to determine the sets $\pi$ such that $G$ has a Hall $\pi$-subgroup.

It is straightforward to modify the method of $\S 7.2$ so that it only checks whether $G$ has a Hall $\pi$-subgroup for a given $\pi$.

To determine all prime number sets $\pi$, note first that it is sufficient to consider those sets $\pi$ with $\pi \subseteq\|G\|$ and $|\pi| \geqslant 2$. Further, for the sets with $2 \notin \pi$, it is sufficient to determine the maximal sets $\pi$. For the sets with $2 \in \pi$, all possible sets need to be considered.

\section{Implementation and runtime}

The algorithm as described here has been implemented by the second author in the computer algebra system GAP [10] and will be made available as part of a forthcoming package. The implementation applies to any group for which the natural homomorphism $G \rightarrow G / \operatorname{Rad}(G)$ and a polycyclic generating set for $\operatorname{Rad}(G)$ can be constructed effectively. Thus it applies, in particular, to all finite permutation or matrix groups. The implementation uses the algorithm of $\S 6.4$ to determine Hall subgroups of simple composition factors.

Table 3 gives some example timings (seconds on a $2.66 \mathrm{MHz}$ Xeon 'Woodcrest' firstgeneration Mac Pro) for our implementation. The columns of the table contain the following information:

- Deg, the permutation- or matrix-group degree of the group;

- $q$, the prime in the case of matrix groups (thus, in particular, the groups for which no $q$ is given are permutation groups);

- $\pi$, the set of primes being considered;

- $n$, the number of conjugacy classes of Hall $\pi$-subgroups;

- $t_{\text {Init }}$, the time required for setting up the required data structures (including determination of the homomorphism $G \rightarrow G / \operatorname{Rad}(G)$ as well as a polycyclic generating set for $\operatorname{Rad}(G)$ ) for calculations with $G$;

- $t_{\text {Run }}$, the time for the actual Hall subgroup computation after all data structures have been set up.

The timings show that runtime is dominated by the set-up (determining radical factors etc.) as well as by calculations of the simple leaves. 
TABLE 3. Example timings for our algorithm.

\begin{tabular}{|c|c|c|c|c|c|c|c|}
\hline Group & Order & Deg & $q$ & $\pi$ & $n$ & $t_{\text {Init }}$ & $t_{\text {Run }}$ \\
\hline $2^{1+8} \cdot\left(\right.$ Alt $_{5} \times$ Alt $\left._{5}\right) \cdot 2$ & $2^{15} 3^{2} 5^{3}$ & 760 & 2 & 2,3 & 1 & 140 & 6 \\
\hline $\mathrm{PSL}_{5}(2)$ & $2^{11} 3^{2} 5^{2} 7 \cdot 31$ & 31 & & $2,3,7$ & 2 & 0 & 4 \\
\hline $\mathrm{M}_{24}$ & $2^{11} 3^{3} 5^{2} 7 \cdot 11 \cdot 23$ & 24 & & $2,3,5$ & 1 & 0 & 11 \\
\hline $\mathrm{PSU}_{3}(13)$ & $2^{5} 3 \cdot 5 \cdot 7^{2} 13^{3} 157$ & 2198 & & 2,3 & 2 & 41 & 161 \\
\hline $\mathrm{PSU}_{3}(13)$ & $2^{5} 3 \cdot 5 \cdot 7^{2} 13^{3} 157$ & 2198 & & 3,13 & 1 & 4 & 1 \\
\hline $2^{1+12} \cdot 3 \cdot \mathrm{M}_{22}: 2$ & $2^{22} 3^{3} 5^{2} 7 \cdot 11$ & 112 & 2 & 5,11 & 1 & 5.3 & 0.7 \\
\hline $2^{1+12} \cdot 3 \cdot \mathrm{M}_{22}: 2$ & $2^{22} 3^{3} 5^{2} 7 \cdot 11$ & 112 & 2 & $2,3,5$ & 1 & 5 & 2 \\
\hline $2^{11} \cdot \mathrm{M}_{24}$ & $2^{22} 3^{3} 5^{2} 7 \cdot 11 \cdot 23$ & 24 & 2 & 5,11 & 1 & 6.5 & 13.5 \\
\hline $2^{11} \cdot \mathrm{M}_{24}$ & $2^{22} 3^{3} 5^{2} 7 \cdot 11 \cdot 23$ & 24 & 2 & $2,3,5$ & 1 & 7.5 & 14 \\
\hline $\mathrm{SL}_{2}(7) \succ \mathrm{S}_{5}$ & $2^{24} 3^{6} 5^{2} 7^{5}$ & 80 & & 2,3 & 4 & 1.3 & 3.7 \\
\hline $\mathrm{GL}_{3}(47)$ & $2^{8} 3 \cdot 5 \cdot 23^{3} 37 \cdot 47^{3} 61$ & 3 & 47 & 23,47 & 1 & 6.6 & 2.7 \\
\hline $\mathrm{PSL}_{3}(2) \imath_{7} \mathrm{PSL}_{3}(2)$ & $2^{25} 3^{8} 5 \cdot 7^{8}$ & 49 & & 3,7 & 1 & 1.3 & 0.7 \\
\hline $\mathrm{PSL}_{3}(2) \imath_{7} \mathrm{PSL}_{3}(2)$ & $2^{25} 3^{8} 5 \cdot 7^{8}$ & 49 & & 2,3 & 8 & 1 & 13 \\
\hline $\mathrm{SL}_{2}(7)$ ? $\mathrm{S}_{6}$ & $2^{29} 3^{8} 5^{2} 7^{6}$ & 96 & & 3,7 & 1 & 2.3 & 0.7 \\
\hline $\mathrm{SL}_{2}(7) \succ \mathrm{S}_{6}$ & $2^{29} 3^{8} 5^{2} 7^{6}$ & 96 & & $2,3,5$ & 2 & 2.5 & 7.5 \\
\hline $\mathrm{SL}_{2}(7)>\mathrm{S}_{6}$ & $2^{29} 3^{8} 5^{2} 7^{6}$ & 14 & 7 & 3,7 & 1 & 7.4 & 0.6 \\
\hline $\mathrm{SL}_{2}(7)>\mathrm{S}_{6}$ & $2^{29} 3^{8} 5^{2} 7^{6}$ & 14 & 7 & $2,3,5$ & 2 & 7 & 5 \\
\hline $\mathrm{S}_{5} \imath_{8} \mathrm{PSL}_{2}(7)$ & $2^{28} 3^{9} 5^{9} 7$ & 40 & & 3,7 & 1 & 18 & 0.6 \\
\hline $\mathrm{S}_{5} \imath_{8} \mathrm{PSL}_{2}(7)$ & $2^{28} 3^{9} 5^{9} 7$ & 40 & & 2,3 & 2 & 16.9 & 3.6 \\
\hline $3^{15}:\left(\mathrm{M}_{11}<\mathrm{S}_{3}\right)$ & $2^{14} 3^{22} 5^{4} 11^{3}$ & 16 & 3 & 5,11 & 1 & 7 & 0.3 \\
\hline $\mathrm{SL}_{2}(7)>\mathrm{S}_{7}$ & $2^{33} 3^{9} 5^{2} 7^{8}$ & 112 & & $2,3,5$ & 4 & 3 & 68 \\
\hline $\mathrm{SL}_{2}(7) \succ \mathrm{S}_{7}$ & $2^{33} 3^{9} 5^{2} 7^{8}$ & 14 & 7 & $2,3,5$ & 4 & 10 & 14 \\
\hline $7^{12}:\left(\mathrm{GL}_{3}(7) \times \operatorname{SP}(4,7)\right)$ & $2^{16} 3^{6} 5^{3} 7^{19} 19$ & 7 & 7 & 5,19 & 1 & 50.3 & 0.7 \\
\hline $2^{44}:\left(\operatorname{PSL} 2(59) \times \mathrm{M}_{23}\right)$ & $2^{56} 3^{5} 5^{4} 7 \cdot 11 \cdot 23 \cdot 29 \cdot 59$ & 42 & 4 & $\begin{array}{r}11,23 \\
29,59\end{array}$ & 1 & 60 & 29 \\
\hline $23^{9}:\left(\mathrm{GL}_{3}(23) \times \mathrm{GL}_{3}(23)\right)$ & $2^{13} 3^{2} 5 \cdot 7^{2} 11^{6} 23^{15} 79^{2}$ & 6 & 23 & 11,23 & 1 & 168 & 6 \\
\hline
\end{tabular}

Acknowledgements. Part of this work was done during the authors' visit to the University of Auckland, whose hospitality is gratefully acknowledged. The first author was supported by the Alexander von Humboldt foundation and the University of Auckland during the visit. We are grateful to the referee for suggesting multiple improvements in the presentation and to Kenneth Monks for language correction.

\section{References}

1. L. BABAi and R. Beals, 'A polynomial-time theory of black box groups I', Groups St Andrews 1997 in Bath, I, London Mathematical Society Lecture Note Series 260 (eds C. M. Campbell, E. F. Robertson, N. Ruskuc and G. C. Smith; Cambridge University Press, Cambridge, 1999) 30-64.

2. J. Cannon, B. Cox and D. Holt, 'Computing Sylow subgroups in permutation groups', J. Symbolic Comput. 24 (1997) no. 3/4, 303-316.

3. J. Cannon, B. Cox and D. Holt, 'Computing the subgroup lattice of a permutation group', J. Symbolic Comput. 31 (2001) no. 1/2, 149-161.

4. J. J. CANnon and D. F. Holt, 'Automorphism group computation and isomorphism testing in finite groups', J. Symbolic Comput. 35 (2003) no. 3, 241-267.

5. J. Cannon, D. Holt, M. Slattery and A. Steel, 'Computing subgroups of bounded index in a finite group', J. Symbolic Comput. 40 (2005) no. 2, 1013-1022.

6. J. J. Cannon, B. Eick and C. R. Leedham-Green, 'Special polycyclic generating sequences for finite soluble groups', J. Symbolic Comput. 38 (2004) no. 5, 1445-1460.

7. F. Celler, J. Neubüser and C. R. B. Wright, 'Some remarks on the computation of complements and normalizers in soluble groups', Acta Appl. Math. 21 (1990) 57-76.

8. W. Feit, 'Some consequences of the classification of finite simple groups', The Santa Cruz Conference on Finite Groups (University of California, Santa Cruz, 1979), Proceedings of Symposia in Pure Mathematics 37 (American Mathematical Society, Providence, RI, 1980) 175-181.

9. W. Feit and J. Thompson, 'Solvability of groups of odd order', Pacific J. Math. 13 (1963) 775-1029.

10. The GAP group, 'GAP - groups, algorithms and programming, version 4.4', 2004, http://www.gap-system.org. 
11. F. Gross, 'Conjugacy of odd order Hall subgroups', Bull. Lond. Math. Soc. 19 (1987) no. 4, 311-319.

12. F. Gross, 'Hall subgroups of order not divisible by 3', Rocky Mountain J. Math. 23 (1993) no. 2, 569-591.

13. F. Gross, 'Conjugacy of odd order Hall subgroups of the classical linear groups', Math. Z. 220 (1995) no. 3, 317-336.

14. P. Hall, 'Theorems like Sylow's', Proc. London Math. Soc. 6 (1956) 286-304.

15. D. F. Holt, 'Representing quotients of permutation groups', Quart. J. Math. Oxford Ser. (2) 48 (1997) no. $191,347-350$.

16. D. F. Holt, B. Eick and E. A. O'Brien, Handbook of computational group theory, Discrete Mathematics and Its Applications (Chapman \& Hall/CRC, Boca Raton, FL, 2005).

17. A. Hulpke, 'Conjugacy classes in finite permutation groups via homomorphic images', Math. Comp. 69 (2000) no. 232, 1633-1651.

18. A. Hulpke, 'Computing conjugacy classes of elements in matrix groups', in preparation, 2012.

19. B. HupperT, Endliche Gruppen I, Grundlehren der mathematischen Wissenschaften 134 (Springer, Berlin, 1967).

20. W. Kantor, 'Sylow's theorem in polynomial time', J. Comput. System Sci. 30 (1985) no. 3, 359-394.

21. W. Kantor, 'Finding Sylow normalizers in polynomial time', J. Algorithms 11 (1990) no. 4, 523-563.

22. E. M. LuKS and Á. SEREss, 'Computing the Fitting subgroup and solvable radical for small-base permutation groups in nearly linear time', Proceedings of the 2nd DIMACS Workshop held at Rutgers University, New Brunswick, NJ, June 7-10, 1995, DIMACS: Series in Discrete Mathematics and Theoretical Computer Science 28 (eds Larry Finkelstein and William M. Kantor; American Mathematical Society, Providence, RI, 1997) 169-181.

23. E. A. O'Brien, 'Algorithms for matrix groups', Groups St Andrews 2009 in Bath. Volume 2, London Mathematical Society Lecture Note Series 388 (eds C. M. Campbell, M. R. Quick, E. F. Robertson, C. M. Roney-Dougal, G. C. Smith and G. Traustason; Cambridge University Press, Cambridge, 2011) 297-323.

24. Robinson, A course in the theory of groups, 2nd edn (Springer, New York, 1996).

25. B. Schmalz, 'Verwendung von Untergruppenleitern zur Bestimmung von Doppelnebenklassen', Bayreuth. Math. Schr. 31 (1990) 109-143.

26. M. Stather, 'Constructive Sylow theorems for the classical groups', J. Algebra 316 (2007) no. 2, 536-559.

27. E. P. VDovin and D. O. Revin, 'Hall subgroups of odd order in finite groups', Algebra Logika 41 (2002) no. $1,8-29$.

28. D. O. REvin and E. P. VDovin, 'On the number of classes of conjugate Hall subgroups in finite simple groups', J. Algebra 324 (2010) no. 12, 3614-3652.

29. D. O. Revin and E. P. VDovin, 'Generalizations of the Sylow theorem', Groups St Andrews 2009 in Bath. Volume 2, London Mathematical Society Lecture Note Series 388 (eds Campbell, C. M. Quick, M. R. Robertson, E. F. Roney-Dougal, C. M. Smith and G. C. Traustason; Cambridge University Press, Cambridge, 2011) 488-519.

30. J. G. Thompson, 'Hall subgroups of the symmetric groups', J. Combin. Theory 1 (1966) 271-279.

Bettina Eick
Institut Computational Mathematics
TU Braunschweig
D-38106 Braunschweig
Germany

beick@tu-bs.de

\author{
Alexander Hulpke \\ Department of Mathematics \\ Colorado State University \\ Fort Collins, CO 80523 \\ USA
}

hulpke@math.colostate.edu 